\title{
Best constants in the exceptional case of Sobolev inequalities
}

\section{Journal Article}

Author(s):

Faget, Zoé

Publication date:

2006-01

Permanent link:

https://doi.org/10.3929/ethz-b-000030940

Rights / license:

In Copyright - Non-Commercial Use Permitted

Originally published in:

Mathematische Zeitschrift 252(1), https://doi.org/10.1007/s00209-005-0850-5 
DOI: $10.1007 / \mathrm{s} 00209-005-0850-5$

\section{Best constants in the exceptional case of Sobolev inequalities}

\section{Zoé Faget}

ETH-Zürich, Departement Mathematik, HG J 65 Rämistrasse 101, CH-8092 Zürich (e-mail: zoe.faget@math.ethz.ch)

Received: 5 January 2004 / Published online: 16 August 2005 - (C) Springer-Verlag 2005

Abstract. We prove the existence of a second best constant in the exceptional case of Sobolev inequalities on a compact Riemannian $n$-manifold locally conformally flat.

Mathematics Subject Classification (2000): 46E35

\section{Introduction}

Sobolev embeddings and inequalities have been the subject of many studies in the past years. Best constant in Sobolev inequalities are fundamental in the study of non linear PDEs on manifolds.

Let $(M, g)$ a Riemannian $n$-manifold, $H_{1}^{q}(M)$ stands for the standard Sobolev space of $L^{q}$-functions with their gradients also in $L^{q}(M)$. The embedding $H_{1}^{q}(M) \subset L^{q n /(n-q)}(M)$ is continuous for $\left.q \in\right] 1, n[$, and we get the existence of $A, B>0$ two constants such that for any $u \in H_{1}^{q}(M)$

$$
\|u\|_{q n /(n-q)}^{q} \leq A\|\nabla u\|_{q}^{q}+B\|u\|_{q}^{q}
$$

The "best constant problem" consists in finding the smallest $A$ possible such that the above inequality remains true for any $u \in H_{1}^{q}(M)$. Knowing the precise value of this best constant allow us to solve non linear PDEs. This problem is now completely solved: in 1974 Aubin established the value of this best constant. Precisely, we have that for any $\varepsilon>0$, there exists $B_{\varepsilon}$ such that for any $u \in H_{1}^{q}(M)$

$$
\|u\|_{q n /(n-q)}^{q} \leq\left(K^{q}(n, q)+\varepsilon\right)\|\nabla u\|_{q}^{q}+B_{\varepsilon}\|u\|_{q}^{q}
$$

where

$$
K(n, q)=\frac{q-1}{n-q}\left(\frac{n-q}{n(q-1)}\right)^{1 / q}\left(\frac{\Gamma(n+1)}{\Gamma(n / q) \Gamma(n+1-n / q) w_{n-1}}\right)^{1 / n}
$$


$w_{n-1}$ being the volume of the standard unit sphere $S_{n-1}$. First Hebey and Vaugon, then Druet proved that it was possible to take $\varepsilon=0$ in the previous inequality.

When $q=n$, one could hope that $H_{1}^{n}(M) \subset L^{\infty}(M)$, but this is not true. However, when $u \in H_{1}^{n}(M)$ we have $e^{u} \in L^{1}(M)$. We then have another Sobolev inequality which can be seen as an extension of traditional Sobolev inequalities to this case, therefore called "exceptional case". Precisely : there exists 3 constants $v, \mu, C>0$ such that for any $u \in H_{1}^{n}(M)$

$$
\int_{M} e^{u} \mathrm{dV} \leq C \exp \left[\mu\|\nabla u\|_{n}^{n}+v\|u\|_{n}^{n}\right]
$$

Once again, the "best constant" is the smallest $\mu$ such that inequality (1) is true for any $u \in H_{1}^{n}(M)$. Cherrier [4] proved that for any $\varepsilon>0$, there exists $A_{\varepsilon}, C_{\varepsilon}>0$ such that for any $u \in H_{1}^{n}(M)$

$$
\int_{M} e^{u} \mathrm{dV} \leq C_{\varepsilon} \exp \left[\left(\mu_{n}+\varepsilon\right)\|\nabla u\|_{n}^{n}+A_{\varepsilon}\|u\|_{n}^{n}\right]
$$

where $\mu_{n}=(n-1)^{n-1} n^{1-2 n} w_{n-1}^{-1}$, and $\mu_{n}$ is the smallest $\mu$ possible such that (2) remains true for any $u \in H_{1}^{n}(M)$. Aubin [2] then proved that for any $\varepsilon>0$, there exists $C_{\varepsilon}>0$ such that for any $u \in H_{1}^{n}(M)$ with $\int_{M} u \mathrm{dV}=0$

$$
\int_{M} e^{u} \mathrm{dV} \leq C_{\varepsilon} \exp \left[\left(\mu_{n}+\varepsilon\right)\|\nabla u\|_{n}^{n}\right]
$$

This result is a better result in the sense that (3) implies (2). Once again, there are applications to the resolution of non-linear elliptic PDEs, this time involving $e^{u}$.

In this article, we prove that it is possible to take $\varepsilon=0$ in (3) (hence in (2)) when $M$ is locally conformally flat. This introduces the notion of a second best constant, precisely the smallest constant $C$ such that for any $u \in H_{1}^{n}(M)$ with $\int_{M} u \mathrm{dV}=0$

$$
\int_{M} e^{u} \mathrm{dV} \leq C \exp \left[\mu_{n}\|\nabla u\|_{n}^{n}\right]
$$

and asks the question of extremal functions, which gives to solutions of PDEs that couldn't be studied to this point. Also, we will note that Hebey and Vaugon [16] have also studied the case when $M$ is locally conformally flat for classical Sobolev inequalities, but the exceptional case of Sobolev inequalities use (by necessity) very different technics. For example, our proof uses a second derivative in the variational problem, which is of no use in the classical case where the functionals are homogeneous. Also, a crucial step of the proof is a rather unusual way of using test functions with a negative exponent.

\section{A first result in the exceptional case of Sobolev inequalities}

Since no confusion is possible, through this whole article we will write $H_{1}^{n}$ for $H_{1}^{n}(M)$ and $L^{q}$ for $L^{q}(M)$. 
Proposition 1. Let $(M, g)$ be a Riemannian n-manifold locally conformally flat. There exist two constants $C_{1}, C_{2}$ such that, for any $u \in H_{1}^{n}$

$$
\int_{M} e^{u} \mathrm{dV} \leq C_{1} \exp \left[\mu_{n}\|\nabla u\|_{n}^{n}+C_{2}\|u\|_{n}^{n}\right]
$$

where $\mu_{n}=(n-1)^{n-1} n^{1-2 n} w_{n-1}^{-1}, w_{n-1}$ being the volume of the standard unit sphere $S_{n}$.

Proof. We use a proof by contradiction. We assume that for any $\alpha, C_{\beta}>0$ there exists $v_{\alpha, \beta}=v_{\alpha} \in H_{1}^{n}$ such that

$$
\int_{M} e^{v_{\alpha}} \mathrm{dV}>C_{\beta} \exp \left[\mu_{n}\left\|\nabla v_{\alpha}\right\|_{n}^{n}+\alpha\left\|v_{\alpha}\right\|_{n}^{n}\right]
$$

which means that for any $\alpha, \beta\left(\beta=\ln C_{\beta}\right)$

$$
\ln \left(\int_{M} e^{v_{\alpha}} \mathrm{dV}\right)>\beta+\mu_{n}\left\|\nabla v_{\alpha}\right\|_{n}^{n}+\alpha\left\|v_{\alpha}\right\|_{n}^{n}
$$

For any $\alpha>0$, we consider the functional $I_{\alpha}$ such that for any $u \in H_{1}^{n}$

$$
I_{\alpha}(u)=\frac{\mu_{n} \int_{M}|\nabla u|^{n} \mathrm{dV}+\alpha \int_{M}|u|^{n} \mathrm{dV}+\beta}{\ln \int_{M} e^{u} \mathrm{dV}}
$$

Let $\lambda_{\alpha}=i n f_{u \in H} I_{\alpha}(u)$, where $H=\left\{u \in H_{1}^{n} \mid \int_{M} e^{u} \mathrm{dV}>1\right\}$. We have $0<\lambda_{\alpha}<$ 1 , and standard variational techniques give the existence of $u_{\alpha} \in H_{1}^{n}$ such that $I_{\alpha}\left(u_{\alpha}\right)=\lambda_{\alpha}$. We note that, since for any $u \in H_{1}^{n}, I_{\alpha}(|u|) \leq I_{\alpha}(u)$, we can choose $u_{\alpha} \geq 0$. We then get

$$
\mu_{n} \int_{M}\left|\nabla u_{\alpha}\right|^{n} \mathrm{dV}+\alpha \int_{M} u_{\alpha}^{n} \mathrm{dV}+\beta=\lambda_{\alpha} \ln \int_{M} e^{u_{\alpha}} \mathrm{dV}
$$

For any $\phi \in H_{1}^{n}$ we have

$$
I_{\alpha, u}^{\prime}(\phi)=n \mu_{n} \int_{M}\left|\nabla u_{\alpha}\right|^{n-2} \nabla u_{\alpha} \nabla \phi \mathrm{dV}+\alpha n \int_{M} u_{\alpha}^{n-1} \phi \mathrm{dV}-\lambda_{\alpha} \frac{\int_{M} e^{u_{\alpha}} \phi \mathrm{dV}}{\int_{M} e^{u_{\alpha}} \mathrm{dV}}(6)
$$

Since $u_{\alpha}$ is a minimizer for $I_{\alpha}$, we get for any $\phi \in H_{1}^{n}, I_{\alpha, u_{\alpha}}^{\prime}(\phi)=0$ and $I_{\alpha, u_{\alpha}}^{\prime \prime}(\phi, \phi) \geq 0$, which gives, after calculations

$$
\begin{aligned}
\lambda_{\alpha} \frac{\int_{M} e^{u_{\alpha}} \phi^{2} \mathrm{dV}}{\int_{M} e^{u_{\alpha}} \mathrm{dV}} \leq & \mu_{n} n(n-1) \int_{M}\left|\nabla u_{\alpha}\right|^{n-2}|\nabla \phi|^{2} \mathrm{dV}+ \\
& +\alpha n(n-1) \int_{M} u_{\alpha}^{n-2} \phi^{2} \mathrm{dV}+\lambda_{\alpha}\left(\frac{\int_{M} e^{u_{\alpha}} \phi \mathrm{dV}}{\int_{M} e^{u_{\alpha}} \mathrm{dV}}\right)^{2}
\end{aligned}
$$

On the other hand, we easily prove (using the fact that $M$ is compact) that for any $\delta>0$, there exists $B$ a ball of radius $\delta$, a real number $\varepsilon>0$, and $\left(u_{\alpha i}\right)$ a subsequence of $\left(u_{\alpha}\right)$ such that

$$
\limsup _{\alpha i \rightarrow+\infty} \frac{\int_{B} e^{u_{\alpha i}} \mathrm{dV}}{\int_{M} e^{u_{\alpha i}} \mathrm{dV}} \geq \varepsilon
$$


We now choose a ball $B$ such that (8) is true on $B, B$ of radius $\delta$ small enough such that $(B(2 \delta), g)$ is conformally isometric to the Eucledean ball (we recall that $M$ is locally conformally flat). We go back to (7) in which we write $\phi=$ $\eta^{n / 2}\left(u_{\alpha}+a\right)^{1 / 2}$, where $a>0$ (later stated more precisely) and we recall that $u_{\alpha} \geq 0$, and $\eta$ is a $\mathcal{C}^{\infty}$ cut-off function, $\eta=1$ on $M \backslash B(2 \delta), \eta=0$ on $B, \eta \leq 1$. We get

$$
\begin{aligned}
\lambda_{\alpha} & \frac{\int_{M} \eta^{n}\left(u_{\alpha}+a\right) e^{u_{\alpha}} \mathrm{dV}}{\int_{M} e^{u_{\alpha}} \mathrm{dV}} \\
\leq & \mu_{n} n(n-1) \int_{M}\left|\nabla u_{\alpha}\right|^{n-2} \mid \nabla\left(\left.\eta^{n / 2}\left(u_{\alpha}+a\right)^{1 / 2}\right|^{2} \mathrm{dV}\right. \\
& \quad+\alpha n(n-1) \int_{M} u_{\alpha}^{n-2} \eta^{n}\left(u_{\alpha}+a\right) \mathrm{dV}+\lambda_{\alpha}\left(\frac{\int_{M} \eta^{n / 2}\left(u_{\alpha}+a\right)^{1 / 2} e^{u_{\alpha}} \mathrm{dV}}{\int_{M} e^{u_{\alpha}} \mathrm{dV}}\right)^{2}
\end{aligned}
$$

In all that follows, we write $C$ for a constant independant of $\alpha$. We have

$$
\left|\nabla\left(\eta^{n / 2}\left(u_{\alpha}+a\right)^{1 / 2}\right)\right| \leq\left(u_{\alpha}+a\right)^{1 / 2}\left|\nabla\left(\eta^{n / 2}\right)\right|+\eta^{n / 2}\left|\nabla\left(u_{\alpha}+a\right)^{1 / 2}\right|
$$

which leads to

$$
\begin{aligned}
\left|\nabla\left(\eta^{n / 2}\left(u_{\alpha}+a\right)^{1 / 2}\right)\right|^{2} & \leq C\left(u_{\alpha}+a\right)\left|\nabla\left(\eta^{n / 2}\right)\right|^{2}+C \eta^{n}\left|\nabla\left(u_{\alpha}+a\right)^{1 / 2}\right|^{2} \\
& \leq C\left(u_{\alpha}+a\right) \eta^{n-2}|\nabla \eta|^{2}+C \eta^{n}\left(u_{\alpha}+a\right)^{-1}\left|\nabla u_{\alpha}\right|^{2}
\end{aligned}
$$

This way

$$
\begin{aligned}
& \int_{M}\left|\nabla u_{\alpha}\right|^{n-2} \mid \nabla\left(\left.\eta^{n / 2}\left(u_{\alpha}+a\right)^{1 / 2}\right|^{2} \mathrm{dV}\right. \\
& \quad \leq C \int_{M}\left|\nabla u_{\alpha}\right|^{n-2}\left(u_{\alpha}+a\right) \eta^{n-2}|\nabla \eta|^{2} \mathrm{dV}+C \int_{M}\left|\nabla u_{\alpha}\right|^{n} \eta^{n}\left(u_{\alpha}+a\right)^{-1} \mathrm{dV}
\end{aligned}
$$

On one hand we have, for any $\varepsilon>0$, there exists $C_{\varepsilon}$ such that $\left|\nabla u_{\alpha}\right|^{n-2} \eta^{n-2}\left(u_{\alpha}+\right.$ a) $|\nabla \eta|^{2} \leq \varepsilon\left(\eta\left|\nabla u_{\alpha}\right|\right)^{n}+C_{\varepsilon}\left(u_{\alpha}+a\right)^{n / 2}|\nabla \eta|^{n}$. On the other hand, we have that $u_{\alpha} \geq 0$, which means that $\left(u_{\alpha}+a\right)^{-1} \leq a^{-1}$. We then get,

$$
\begin{aligned}
& \int_{M}\left|\nabla u_{\alpha}\right|^{n-2} \mid \nabla\left(\left.\eta^{n / 2}\left(u_{\alpha}+a\right)^{1 / 2}\right|^{2} \mathrm{dV}\right. \\
& \quad \leq\left(\frac{C}{a}+\varepsilon\right) \int_{M} \eta^{n}\left|\nabla u_{\alpha}\right|^{n} \mathrm{dV}+C_{\varepsilon} \int_{M}|\nabla \eta|^{n}\left(u_{\alpha}+a\right)^{n / 2} \mathrm{dV}
\end{aligned}
$$

We also have $\left(u_{\alpha}+a\right)^{n / 2} \leq C\left(u_{\alpha}^{n / 2}+a^{n / 2}\right) \leq C u_{\alpha}^{n / 2}+C$ and $|\nabla \eta| \leq C$ on $M$ which gives

$$
\int_{M}|\nabla \eta|^{n}\left(u_{\alpha}+a\right)^{n / 2} \mathrm{dV} \leq C \int_{M} u_{\alpha}^{n / 2} \mathrm{dV}+C
$$

Since $u_{\alpha} \in H_{1}^{n},\left\|u_{\alpha}\right\|_{n / 2} \leq C$, and this leads to, with all the previous,

$$
\int_{M}\left|\nabla u_{\alpha}\right|^{n-2} \mid \nabla\left(\left.\eta^{n / 2}\left(u_{\alpha}+a\right)^{1 / 2}\right|^{2} \mathrm{dV} \leq\left(\frac{C}{a}+\varepsilon\right) \int_{M} \eta^{n}\left|\nabla u_{\alpha}\right|^{n} \mathrm{dV}+C_{\varepsilon}\right.
$$


Since $\eta=0$ on $B$, we also have

$$
\int_{M} \eta^{n / 2}\left(u_{\alpha}+a\right)^{1 / 2} e^{u_{\alpha}} \mathrm{dV}=\int_{M \backslash B} \eta^{n / 2}\left(u_{\alpha}+a\right)^{1 / 2} e^{u_{\alpha}} \mathrm{dV}
$$

and by Hölder's inequality

$$
\int_{M} \eta^{n / 2}\left(u_{\alpha}+a\right)^{1 / 2} e^{u_{\alpha}} \mathrm{dV} \leq\left(\int_{M \backslash B} \eta^{n}\left(u_{\alpha}+a\right) e^{u_{\alpha}} \mathrm{dV}\right)^{1 / 2}\left(\int_{M \backslash B} e^{u_{\alpha}} \mathrm{dV}\right)^{1 / 2}
$$

We then get, together with (8), up to a subsequence,

$$
\begin{aligned}
\left(\frac{\int_{M \backslash B} \eta^{n / 2}\left(u_{\alpha}+a\right)^{1 / 2} e^{u_{\alpha}} \mathrm{dV}}{\int_{M} e^{u_{\alpha}} \mathrm{dV}}\right)^{2} & \leq \frac{\int_{M \backslash B} \eta^{n} e^{u_{\alpha}}\left(u_{\alpha}+a\right) \mathrm{dV}}{\int_{M} e^{u_{\alpha}} \mathrm{dV}} \frac{\int_{M \backslash B} e^{u_{\alpha}} \mathrm{dV}}{\int_{M} e^{u_{\alpha}} \mathrm{dV}} \\
& \leq(1-c) \frac{\int_{M} \eta^{n} e^{u_{\alpha}}\left(u_{\alpha}+a\right) \mathrm{dV}}{\int_{M} e^{u_{\alpha}} \mathrm{dV}}
\end{aligned}
$$

with $c>0$. From the previous, we get

$$
\begin{gathered}
\lambda_{\alpha} \frac{\int_{M} \eta^{n}\left(u_{\alpha}+a\right) e^{u_{\alpha}} \mathrm{dV}}{\int_{M} e^{u_{\alpha}} \mathrm{dV}}-\lambda_{\alpha}\left(\frac{\int_{M} \eta^{n / 2}\left(u_{\alpha}+a\right)^{1 / 2} e^{u_{\alpha}} \mathrm{dV}}{\int_{M} e^{u_{\alpha}} \mathrm{dV}}\right)^{2} \\
\geq \lambda_{\alpha} c \frac{\int_{M} \eta^{n} e^{u_{\alpha}}\left(u_{\alpha}+a\right) \mathrm{dV}}{\int_{M} e^{u_{\alpha}} \mathrm{dV}}
\end{gathered}
$$

and $c>0$. Since $u_{\alpha} \in H_{1}^{n}$, we easily bound the last bit of (9),

$$
\begin{gathered}
\alpha C \int_{M} u_{\alpha}^{n-2} \eta^{n}\left(u_{\alpha}+a\right) \mathrm{dV} \leq \alpha C \int_{M \backslash B} u_{\alpha}^{n-2}\left(u_{\alpha}+a\right) \mathrm{dV} \\
\leq \alpha C \int_{M \backslash B} u_{\alpha}^{n-1} \mathrm{dV}+C \alpha a \int_{M \backslash B} u_{\alpha}^{n-2} \mathrm{dV} \leq C \alpha
\end{gathered}
$$

We get back to (9) together with (10), (11) and (12) and we get

$$
\lambda_{\alpha} c \frac{\int_{M} \eta^{n} e^{u_{\alpha}}\left(u_{\alpha}+a\right) \mathrm{dV}}{\int_{M} e^{u_{\alpha}} \mathrm{dV}} \leq\left(\frac{C}{a}+\varepsilon\right) \int_{M} \eta^{n}\left|\nabla u_{\alpha}\right|^{n} \mathrm{dV}+C a \alpha+C_{\varepsilon}
$$

and, since $c>0$ and $u_{\alpha}+a>u_{\alpha}$, this leads to

$$
\lambda_{\alpha} \frac{\int_{M} \eta^{n} e^{u_{\alpha}} u_{\alpha} \mathrm{dV}}{\int_{M} e^{u_{\alpha}} \mathrm{dV}} \leq\left(\frac{C}{a}+\varepsilon\right) \int_{M} \eta^{n}\left|\nabla u_{\alpha}\right|^{n} \mathrm{dV}+C a \alpha+C_{\varepsilon}
$$

In (6), we write $\phi=\eta^{n} u_{\alpha}$ and we get

$$
\lambda_{\alpha} \frac{\int_{M} \eta^{n} e^{u_{\alpha}} u_{\alpha} \mathrm{dV}}{\int_{M} e^{u_{\alpha}} \mathrm{dV}}=n \mu_{n} \int_{M}\left|\nabla u_{\alpha}\right|^{n-2} \nabla\left(\eta^{n} u_{\alpha}\right) \nabla u_{\alpha} \mathrm{dV}+\alpha n \int_{M} u_{\alpha}^{n} \eta^{n} \mathrm{dV}
$$


Since

$$
\begin{aligned}
& \int_{M}\left|\nabla u_{\alpha}\right|^{n-2} \nabla\left(\eta^{n} u_{\alpha}\right) \nabla u_{\alpha} \mathrm{dV} \\
& =\int_{M}\left|\nabla u_{\alpha}\right|^{n} \eta^{n} \mathrm{dV}+n \int_{M} u_{\alpha}\left|\nabla u_{\alpha}\right|^{n-2} \nabla \eta \nabla u_{\alpha} \eta^{n-1} \mathrm{dV}
\end{aligned}
$$

and since for any $\varepsilon>0$ there exists $C_{\varepsilon}$ such that

$$
\left|\nabla u_{\alpha}\right|^{n-1} \eta^{n-1} u_{\alpha}|\nabla \eta| \leq \varepsilon\left|\nabla u_{\alpha}\right|^{n} \eta^{n}+C_{\varepsilon} u_{\alpha}^{n}|\nabla \eta|^{n}
$$

we get that

$$
\begin{aligned}
& n \mu_{n} \int_{M}\left|\nabla u_{\alpha}\right|^{n-2} \nabla\left(\eta^{n} u_{\alpha}\right) \nabla u_{\alpha} \mathrm{dV} \\
& \quad \geq\left(n \mu_{n}-\varepsilon\right) \int_{M}\left|\nabla u_{\alpha}\right|^{n} \eta^{n} \mathrm{dV}-C_{\varepsilon} \int_{M} u_{\alpha}^{n}|\nabla \eta|^{n} \mathrm{dV}
\end{aligned}
$$

and since $\nabla \eta=0, \eta=0$ on $B$

$$
\begin{aligned}
& \lambda_{\alpha} \frac{\int_{M} \eta^{n} e^{u_{\alpha}} u_{\alpha} \mathrm{dV}}{\int_{M} e^{u_{\alpha}} \mathrm{dV}} \\
& \quad=n \mu_{n} \int_{M}\left|\nabla u_{\alpha}\right|^{n-2} \nabla\left(\eta^{n} u_{\alpha}\right) \nabla u_{\alpha} \mathrm{dV}+\alpha n \int_{M} u_{\alpha}^{n} \eta^{n} \mathrm{dV} \\
& \quad \geq\left(n \mu_{n}-\varepsilon\right) \int_{M}\left|\nabla u_{\alpha}\right|^{n} \eta^{n} \mathrm{dV}-C_{\varepsilon} \int_{M \backslash B} u_{\alpha}^{n} \mathrm{dV}
\end{aligned}
$$

Getting back to (13) and (14) we find

$$
\begin{gathered}
\left(n \mu_{n}-\varepsilon\right) \int_{M}\left|\nabla u_{\alpha}\right|^{n} \eta^{n} \mathrm{dV}-C_{\varepsilon} \int_{M \backslash B} u_{\alpha}^{n} \mathrm{dV} \\
\leq\left(\frac{C}{a}+\varepsilon\right) \int_{M} \eta^{n}\left|\nabla u_{\alpha}\right|^{n} \mathrm{dV}+C \alpha+C_{\varepsilon}
\end{gathered}
$$

and finally, choosing $a$ big enough and $\varepsilon$ such that $n \mu_{n}-2 \varepsilon-C / a>0$, we get

$$
\int_{M \backslash B}\left|\nabla u_{\alpha}\right|^{n} \mathrm{dV} \leq C \int_{M \backslash B} u_{\alpha}^{n} \mathrm{dV}+C \alpha+C
$$

We need the following result.

Lemma 1. Let $(M, g)$ a compact Riemannian n-manifold locally conformally flat. For any open ball $B$ of radius $\delta$ small enough there exists $C$ such that for $v \in$ $H_{1,0}^{n}(B)$

$$
\int_{B} e^{v} \mathrm{dV} \leq C \exp \left[\mu_{n} \int_{B}|\nabla v|^{n} \mathrm{dV}\right]
$$

where $H_{1,0}^{n}(B)$ is the closure in $H_{1}^{n}(B)$ of the space of $C^{\infty}$-functions with compact support in $B$. 
Proof. On $(B, \xi) \subset \mathbb{R}^{n}$ we know, thanks to Cherrier [4], that for any $v \in H_{1}^{n}\left(\mathbb{R}^{n}\right)$

$$
\int_{B} e^{v} \mathrm{~d} \xi \leq C \exp \left[\mu_{n} \int_{B}\left|\nabla_{\xi} v\right|^{n} \mathrm{~d} \xi\right]
$$

Since $M$ is locally conformally flat, we can choose a ball $B(\delta) \subset M$ of radius $\delta$ small enough such that on $B(\delta)$, up to an isomorphism, $g=\Phi \xi$, where $\xi$ is the Euclidean metric of $\mathbb{R}^{n}$ and with $\Phi \in \mathcal{C}^{\infty}(B), \Phi>0$. We have $\mathrm{d} \xi=\Phi^{-n / 2} \mathrm{dV}$ and $\left|\nabla_{\xi} v\right|^{n}=\Phi^{n / 2}\left|\nabla_{g} v\right|^{n}$, which leads to, for any $v \in \stackrel{H}{H}_{1}^{n}(B)$

$$
\int_{B} e^{v} \Phi^{-n / 2} \mathrm{dV} \leq C \exp \left[\mu_{n} \int_{B} \Phi^{n / 2}\left|\nabla_{g} v\right|^{n} \Phi^{-n / 2} \mathrm{dV}\right]
$$

and this way, since $\Phi \in \mathcal{C}^{\infty}(B)$, there exists $C$ such that for any $u \in H_{1}^{n}(B)$

$$
\int_{B} e^{v} \mathrm{dV} \leq C \exp \left[\mu_{n} \int_{B}\left|\nabla_{g} v\right|^{n} \mathrm{dV}\right]
$$

We now consider $\zeta$ a $\mathcal{C}^{\infty}$ cut off function, $\zeta=1$ on $B, \zeta=0$ on $M \backslash B(2 \delta), \zeta \leq 1$ on $M$. With Lemma 1 we get

$$
\int_{M} e^{\zeta u_{\alpha}} \mathrm{dV} \leq C \exp \left[\mu_{n} \int_{M}\left|\nabla\left(\zeta u_{\alpha}\right)\right|^{n} \mathrm{dV}\right]
$$

We recall that on the $n$-manifold $M \backslash B$ there exists $C$ such that

$$
\int_{M \backslash B} e^{u_{\alpha}} \mathrm{dV} \leq C \exp \left[C \int_{M \backslash B}\left|\nabla u_{\alpha}\right|^{n} \mathrm{dV}+C \int_{M \backslash B} u_{\alpha}^{n} \mathrm{dV}\right]
$$

We have

$$
\begin{aligned}
& \int_{M} e^{u_{\alpha}} \mathrm{dV}=\int_{M \backslash B} e^{u_{\alpha}} \mathrm{dV}+\int_{B} e^{u_{\alpha}} \mathrm{dV} \\
& \leq C \exp \left[C \int_{M \backslash B}\left|\nabla u_{\alpha}\right|^{n} \mathrm{dV}+C \int_{M \backslash B} u_{\alpha}^{n} \mathrm{dV}\right]+C \int_{M} e^{\zeta u_{\alpha}} \mathrm{dV} \\
& \leq C \exp \left[C \int_{M \backslash B}\left|\nabla u_{\alpha}\right|^{n} \mathrm{dV}+C \int_{M \backslash B} u_{\alpha}^{n} \mathrm{dV}\right] \\
& \quad+C \exp \left[\mu_{n} \int_{M}\left|\nabla\left(\zeta u_{\alpha}\right)\right|^{n} \mathrm{dV}\right]
\end{aligned}
$$

because $M$ is locally conformally flat. We can bound from above

$$
\int_{M}\left|\nabla\left(\zeta u_{\alpha}\right)\right|^{n} \mathrm{dV} \leq \int_{M} \zeta^{n}\left|\nabla u_{\alpha}\right|^{n} \mathrm{dV}+C \int_{M} u_{\alpha}^{n} \mathrm{dV}+C \int_{M \backslash B}\left|\nabla u_{\alpha}\right|^{n} \mathrm{dV}
$$


This leads to, since $\zeta \leq 1$ on $M$,

$$
\begin{aligned}
& \int_{M} e^{u_{\alpha}} \mathrm{dV} \leq C \exp \left[\int_{M \backslash B}\left|\nabla u_{\alpha}\right|^{n} \mathrm{dV}+C \int_{M \backslash B} u_{\alpha}^{n} \mathrm{dV}\right] \\
& +C \exp \left[\mu_{n} \int_{M}\left|\nabla u_{\alpha}\right|^{n} \mathrm{dV}+C \int_{M \backslash B}\left|\nabla u_{\alpha}\right|^{n} \mathrm{dV}+C \int_{M} u_{\alpha}^{n} \mathrm{dV}\right] \\
& \leq C \exp \left[\mu_{n} \int_{M}\left|\nabla u_{\alpha}\right|^{n} \mathrm{dV}+C \int_{M \backslash B}\left|\nabla u_{\alpha}\right|^{n} \mathrm{dV}+C \int_{M} u_{\alpha}^{n} \mathrm{dV}\right]
\end{aligned}
$$

Together with (15) we get

$$
\ln \left(\int_{M} e^{u_{\alpha}} \mathrm{dV}\right) \leq \mu_{n} \int_{M}\left|\nabla u_{\alpha}\right|^{n} \mathrm{dV}+C \int_{M} u_{\alpha}^{n} \mathrm{dV}+C \alpha+C
$$

But, according to (5)

$$
\begin{aligned}
\mu_{n} \int_{M}\left|\nabla u_{\alpha}\right|^{n} \mathrm{dV}+\alpha \int_{M} u_{\alpha}^{n} \mathrm{dV}+\beta & =\lambda_{\alpha} \ln \left(\int_{M} e^{u_{\alpha}} \mathrm{dV}\right) \\
& \leq \ln \left(\int_{M} e^{u_{\alpha}} \mathrm{dV}\right)
\end{aligned}
$$

because $\lambda_{\alpha} \leq 1$. This leads to

$$
\alpha \int_{M} u_{\alpha}^{n} \mathrm{dV}+\beta \leq \bar{C} \int_{M} u_{\alpha}^{n} \mathrm{dV}+C \alpha+C
$$

Since $\alpha$ and $\beta$ can be chosen as wanted, if $\alpha \geq \bar{C}$, we get a contradiction when $\beta$ goes to $+\infty$. This proves the proposition.

\section{A stronger result in the exceptional case of Sobolev inequalities}

We now prove the theorem.

Theorem 1. Let $(M, g)$ be a locally conformally flat $n$-manifold. There exists $C$ such that for any $u \in H_{1}^{n}$ with $\int_{M} u \mathrm{dV}=0$

$$
\int_{M} e^{u} \mathrm{dV} \leq C \exp \left[\mu_{n} \int_{M}|\nabla u|^{n} \mathrm{dV}\right]
$$

where $\mu_{n}=(n-1)^{n-1} n^{1-2 n} w_{n-1}^{-1}, w_{n-1}$ being the volume of the standard unit sphere $S_{n-1}, \mu_{n}$ being the smallest constant possible such that the above inequality remains true for any $u \in H_{1}^{n}, \int_{M} u \mathrm{dV}=0$. In other words, there exists $C$ such that for any $u \in H_{1}^{n}$

$$
\int_{M} e^{u} \mathrm{dV} \leq C \exp \left[\mu_{n} \int_{M}|\nabla u|^{n} \mathrm{dV}+\frac{1}{\operatorname{vol}(M)} \int_{M} u \mathrm{dV}\right]
$$


Proof. Once again, we proceed by contradiction. Assume that for any $C_{\alpha}$, there exists $v_{\alpha} \in H_{1}^{n}$ with $\int_{M} v_{\alpha} \mathrm{dV}=0$ such that

$$
\int_{M} e^{v_{\alpha}} \mathrm{dV}>C_{\alpha} \exp \left[\mu_{n} \int_{M}\left|\nabla v_{\alpha}\right|^{n} \mathrm{dV}\right]
$$

which means that for any $\alpha>0\left(\alpha=\ln C_{\alpha}\right)$

$$
\ln \left(\int_{M} e^{v_{\alpha}} \mathrm{dV}\right)>\alpha+\mu_{n} \int_{M}\left|\nabla v_{\alpha}\right|^{n} \mathrm{dV}
$$

Let $H=\left\{u \in H_{1}^{n} \mid \int_{M} e^{u} \mathrm{dV}>1, \int_{M} u \mathrm{dV}=0\right\}$. We consider the functional $I_{\alpha}$ such that for any $u \in H$

$$
I_{\alpha}(u)=\frac{\alpha+\mu_{n} \int_{M}|\nabla u|^{n} \mathrm{dV}}{\ln \left(\int_{M} e^{u} \mathrm{dV}\right)}
$$

Let $\lambda_{\alpha}=\inf _{u \in H} I_{\alpha}(u)$ With our beginning assumption, we have that $0<\lambda_{\alpha}<1$. We now prove with the variational method that there exists $u_{\alpha} \in H$ such that $I_{\alpha}\left(u_{\alpha}\right)=\lambda_{\alpha}$.

Let $\left(u_{i}\right), u_{i} \in H$ for any $i$, a minimizing sequence for $I_{\alpha}$ (i.e, $I_{\alpha}\left(u_{i}\right)$ goes to $\lambda_{\alpha}$ with $i$ ). For any $u \in H$ we get with Poincare's inequality

$$
\int_{M}|u|^{n} \mathrm{dV} \leq C \int_{M}|\nabla u|^{n} \mathrm{dV}
$$

so in order to prove that $\left(u_{i}\right)$ is bounded in $H_{1}^{n}$, one only has to prove that $\int_{M}\left|\nabla u_{i}\right|^{n} \mathrm{dV}$ is bounded. Since $\lambda_{\alpha}<1$, for a set $\alpha$ there exists $c>0$ such that $\lambda_{\alpha}<1-c$, and since $I_{\alpha}\left(u_{i}\right)$ goes to $\lambda_{\alpha}$, for $i$ big enough there exists $c^{\prime}>0$ such that $I_{\alpha}\left(u_{i}\right) \leq 1-c^{\prime}$. This way, we get

$$
\mu_{n} \int_{M}\left|\nabla u_{i}\right|^{n} \mathrm{dV}+\alpha \leq\left(1-c^{\prime}\right) \ln \left(\int_{M} e^{u_{i}} \mathrm{dV}\right)
$$

On the other hand we know, thanks to Aubin [2] that for any $\varepsilon>0$ there exists $C_{\varepsilon}$ such that for any $u \in H$

$$
\ln \left(\int_{M} e^{u} \mathrm{dV}\right) \leq C_{\varepsilon}+\left(\mu_{n}+\varepsilon\right) \int_{M}|\nabla u|^{n} \mathrm{dV}
$$

Getting back to (16) we get that for any $\varepsilon>0$ there exists $C_{\varepsilon}$ such that

$$
\mu_{n} \int_{M}\left|\nabla u_{i}\right|^{n} \mathrm{dV}+\alpha \leq\left(1-c^{\prime}\right) C_{\varepsilon}+\left(1-c^{\prime}\right)\left(\mu_{n}+\varepsilon\right) \int_{M}\left|\nabla u_{i}\right|^{n} \mathrm{dV}
$$

It is possible to choose $\varepsilon$ such that $\mu_{n}-\left(1-c^{\prime}\right) \mu_{n}-\left(1-c^{\prime}\right) \varepsilon>0$ and we get that there exists $C$ such that $\int_{M}\left|\nabla u_{i}\right|^{n} \mathrm{dV} \leq C$, and $\left(u_{i}\right)$ is bounded in $H_{1}^{n}$. Hence, $\left(u_{i}\right)$ converges weakly in $H_{1}^{n}$ to a function $u_{\alpha}$, strongly in any $L^{q}$ and a.e. We get that $\int_{M} u_{\alpha} \mathrm{dV}=0$ and $\int_{M} e^{u_{\alpha}} \mathrm{dV}>1$. Hence, $u_{\alpha} \in H$. Since $\left(u_{i}\right)$ goes to $u_{\alpha}$ weakly 
in $H_{1}^{n}$ and $\int_{M} e^{u_{i}} \mathrm{dV}$ goes to $\int_{M} e^{u_{\alpha}} \mathrm{dV}$, and since $\left(u_{i}\right)$ is a minimizing sequence for $I_{\alpha}$, we have

$$
\frac{\mu_{n} \int_{M}\left|\nabla u_{\alpha}\right|^{n} \mathrm{dV}+\alpha}{\ln \left(\int_{M} e^{u_{\alpha}} \mathrm{dV}\right)} \leq \lim _{i \rightarrow+\infty} \frac{\mu_{n} \int_{M}\left|\nabla u_{i}\right|^{n} \mathrm{dV}+\alpha}{\ln \left(\int_{M} e^{u_{i}} \mathrm{dV}\right)}=\lambda_{\alpha}
$$

Since $\lambda_{\alpha}=\inf _{u \in H} I_{\alpha}$ and $u_{\alpha} \in H$ this means $I_{\alpha}\left(u_{\alpha}\right)=\lambda_{\alpha}$. Precisely,

$$
\lambda_{\alpha}=\frac{\alpha+\mu_{n} \int_{M}\left|\nabla u_{\alpha}\right|^{n} \mathrm{dV}}{\ln \left(\int_{M} e^{u_{\alpha}} \mathrm{dV}\right)}
$$

We also get, for any $\phi \in H_{1}^{n}, I_{\alpha, u_{\alpha}}^{\prime}(\phi)=\gamma_{\alpha} \int_{M} \phi \mathrm{dV}$, which means, given the expression of $I_{\alpha, u_{\alpha}}^{\prime}$ : for any $\phi \in H_{1}^{n}$

$$
n \mu_{n} \int_{M}\left|\nabla u_{\alpha}\right|^{n-2} \nabla u_{\alpha} \nabla \phi \mathrm{dV}-\lambda_{\alpha} \frac{\int_{M} e^{u_{\alpha}} \phi \mathrm{dV}}{\int_{M} e^{u_{\alpha}} \mathrm{dV}}+\frac{\lambda_{\alpha}}{V} \int_{M} \phi \mathrm{dV}=0
$$

We deduce from Proposition 1 that there exists $C_{1}, C_{2}$ such that, for any $u \in H_{1}^{n}$

$$
\ln \left(\int_{M} e^{u} \mathrm{dV}\right) \leq C_{1}+\mu_{n} \int_{M}|\nabla u|^{n} \mathrm{dV}+C_{2} \int_{M}|u|^{n} \mathrm{dV}
$$

According to (17) and (19), we only have to prove that there exists $C$ (not depending on $\alpha$ ) such that $\left\|u_{\alpha}\right\|_{n}^{n} \leq C$ to get a contradiction. Indeed,

$$
\begin{aligned}
\alpha & \leq \lambda_{\alpha} \ln \left(\int_{M} e^{u_{\alpha}} \mathrm{dV}\right)-\mu_{n} \int_{M}\left|\nabla u_{\alpha}\right|^{n} \mathrm{dV} \text { by }(17) \\
& \leq C+\lambda_{\alpha} \mu_{n} \int_{M}\left|\nabla u_{\alpha}\right|^{n} \mathrm{dV}+C_{2} \int_{M}\left|u_{\alpha}\right|^{n} \mathrm{dV}-\mu_{n} \int_{M}\left|\nabla u_{\alpha}\right|^{n} \mathrm{dV} \text { by (19) } \\
& \leq C+\mu_{n}\left(\lambda_{\alpha}-1\right) \int_{M}\left|\nabla u_{\alpha}\right|^{n} \mathrm{dV}+C_{2} \int_{M}\left|u_{\alpha}\right|^{n} \mathrm{dV}
\end{aligned}
$$

and $\lambda_{\alpha}-1 \leq 0$.

In order to bound $\left\|u_{\alpha}\right\|_{n}$, the idea is to test in (18) $\Phi=u_{\alpha}^{k}$, where $k<0$. Since $u_{\alpha}$ changes sign, we proceed as follows. Let

$$
\begin{aligned}
\Omega_{1} & =\left\{x \in M \mid u_{\alpha}(x) \geq 1\right\} \\
\Omega_{-1} & =\left\{x \in M \mid u_{\alpha}(x) \leq-1\right\} \\
\Omega & =\left\{x \in M|| u_{\alpha}(x) \mid \leq 1\right\}
\end{aligned}
$$

$\left(\Omega_{1}, \Omega_{-1}, \Omega\right.$ depending on $\left.\alpha\right)$, and let $f$ the real function defined by

$$
\begin{aligned}
f(x) & =x^{-1 / n} \quad \text { when } x \geq 1 \\
& =x \quad \text { when }-1 \leq x \leq 1 \\
& =-|x|^{-1 / n} \quad \text { when } x \leq-1
\end{aligned}
$$


The function $f$ is Lipchitzian. We consider $\phi=f \circ u_{\alpha}$. Since $f$ is Lipchitzian and $u_{\alpha} \in H_{1}^{n}$ then $\phi \in H_{1}^{n}$. We then have

$$
\begin{aligned}
\nabla \phi & =-\frac{1}{n} u_{\alpha}^{-(n+1) / n} \nabla u_{\alpha} \text { on } \Omega_{1} \\
& =\nabla u_{\alpha} \text { on } \Omega \\
& =-\frac{1}{n}\left|u_{\alpha}\right|^{-(n+1) / n} \nabla u_{\alpha} \text { on } \Omega_{-1}
\end{aligned}
$$

We get

$$
\int_{M}\left|\nabla u_{\alpha}\right|^{n-2} \nabla u_{\alpha} \nabla \phi \mathrm{dV}=-\frac{1}{n} \int_{\Omega_{1} \cup \Omega_{-1}}\left|u_{\alpha}\right|^{-(n+1) / n}\left|\nabla u_{\alpha}\right|^{n} \mathrm{dV}+\int_{\Omega}\left|\nabla u_{\alpha}\right|^{n} \mathrm{dV}
$$

In (18), we take $\phi=f \circ u_{\alpha}$, we get

$$
\begin{aligned}
& -\mu_{n} \int_{\Omega_{1} \cup \Omega_{-1}}\left|u_{\alpha}\right|^{-(n+1) / n}\left|\nabla u_{\alpha}\right|^{n} \mathrm{dV}+n \mu_{n} \int_{\Omega}\left|\nabla u_{\alpha}\right|^{n} \mathrm{dV} \\
& +\frac{\lambda_{\alpha}}{V} \int_{\Omega_{1}}\left|u_{\alpha}\right|^{-1 / n} \mathrm{dV}-\frac{\lambda_{\alpha}}{V} \int_{\Omega_{-1}}\left|u_{\alpha}\right|^{-1 / n} \mathrm{dV} \\
& \quad+\frac{\lambda_{\alpha}}{V} \int_{\Omega} u_{\alpha} \mathrm{dV}=\lambda_{\alpha} \frac{\int_{M} e^{u_{\alpha}} \Phi \mathrm{dV}}{\int_{M} e^{u_{\alpha}} \mathrm{dV}} \\
& =\lambda_{\alpha} \frac{\int_{\Omega_{1}} e^{u_{\alpha}} u_{\alpha}^{-1 / n} \mathrm{dV}-\int_{\Omega_{-1}} e^{u_{\alpha}}\left|u_{\alpha}\right|^{-1 / n} \mathrm{dV}+\int_{\Omega} e^{u_{\alpha}} u_{\alpha} \mathrm{dV}}{\int_{M} e^{u_{\alpha}} \mathrm{dV}}
\end{aligned}
$$

On $\Omega_{1}, u_{\alpha} \geq 1$ so $u_{\alpha}^{-1 / n} \leq 1$ and then

$$
\frac{\int_{\Omega_{1}} e^{u_{\alpha}} u_{\alpha}^{-1 / n} \mathrm{dV}}{\int_{M} e^{u_{\alpha}} \mathrm{dV}} \leq 1
$$

On $\Omega,\left|u_{\alpha}\right| \leq 1$ so

$$
\frac{\int_{\Omega} e^{u_{\alpha}} u_{\alpha} \mathrm{dV}}{\int_{M} e^{u_{\alpha}} \mathrm{dV}} \leq 1
$$

In fact,

$$
\frac{\int_{M} e^{u_{\alpha}} \phi \mathrm{dV}}{\int_{M} e^{u_{\alpha}} \mathrm{dV}} \leq C
$$

With the same kind of arguments, we easily verify that $\int_{M} \phi \mathrm{dV} \leq C$. This leads to, with (20)

$$
n \mu_{n} \int_{M}\left|\nabla u_{\alpha}\right|^{n-2} \nabla u_{\alpha} \nabla \phi \mathrm{dV} \leq C
$$


The key idea now is to consider the function $v$ such that $|v| \leq 1$ on $M$ defined by

$$
\begin{aligned}
v & =u_{\alpha}^{\left(n^{2}-n-1\right) / n^{2}} \text { on } \Omega_{1} \\
& =u_{\alpha} \text { on } \Omega \\
& =-\left|u_{\alpha}\right|^{\left(n^{2}-n-1\right) / n^{2}} \text { on } \Omega_{-1}
\end{aligned}
$$

We verify that $\int_{M}|\nabla v|^{n} \mathrm{dV} \leq C \int_{M}\left|\nabla u_{\alpha}\right|^{n-2} \nabla u_{\alpha} \nabla \phi \mathrm{dV}$ where $\phi=f \circ u_{\alpha}$ as defined earlier, and by (21), $\int_{M}|\nabla v|^{n} \mathrm{dV} \leq C$. In order to go on with the proof, we need the following Lemma,

Lemma 2. Let $(M, g)$ a Riemannian n-manifold. For any $p \geq 1, q>0$, there exists $C$ such that, for any $u \in H_{1}^{n}$,

$$
\left[\int_{M}|u|^{p} \mathrm{dV}\right]^{n / p} \leq C \int_{M}|\nabla u|^{n} \mathrm{dV}+C\left|\int_{M} h(u) \mathrm{dV}\right|^{q}
$$

Where $h$ is the real function defined by $h(x)=(\operatorname{sign}(x))|x|^{n / q}$

We prove the Lemma.

Proof. We consider the functional $I$ such that for any $u \in H_{1}^{n}$

$$
I(u)=\frac{\int_{M}|\nabla u|^{n} \mathrm{dV}+\left|\int_{M} h(u) \mathrm{dV}\right|^{q}}{\left[\int_{M}|u|^{p} \mathrm{dV}\right]^{n / p}}
$$

Since $I$ is homogeneous, we consider $P=\left\{\left.u \in H_{1}^{n}\left|\int_{M}\right| u\right|^{p} \mathrm{dV}=1\right\}$ and $\mu=$ $\inf _{u \in P} I(u)$. Using the fact that for any $k \geq 1$, the embedding of $H_{1}^{n}$ in $L^{k}$ is compact, standard techniques give the existence of $w \in P$ such that $I(w)=\mu$. It is clear that $\mu \geq 0$, we now want to prove that $\mu>0$. If $\mu=0$, since $I(w)=\mu$ then $\int_{M}|\nabla w| \mathrm{dV}=0$ and $w$ is constant. Since $\int_{M} h(w) \mathrm{dV}=0$ then $w \equiv 0$. But $\int_{M}|w|^{p} \mathrm{dV}=1$ since $v$ is in $P$, which is absurd. This way $\mu>0$, and we proved (22).

We go on with the proof of the Theorem. We apply (22) to $v$ defined above with $q=\left(n^{2}-n-1\right) / n$. We get for any $p \geq 1$

$$
\begin{aligned}
& {\left[\int_{\Omega_{1}} u_{\alpha}^{p\left(n^{2}-n-1\right) / n^{2}} \mathrm{dV}-\int_{\Omega_{-1}}\left|u_{\alpha}\right|^{p\left(n^{2}-n-1\right) / n^{2}} \mathrm{dV}+\int_{\Omega} u_{\alpha}^{p} \mathrm{dV}\right]^{n / p}} \\
& \quad \leq C \int_{M}|\nabla v|^{n} \mathrm{dV}+C\left(\int_{\Omega_{1} \cup \Omega_{-1}} u_{\alpha} \mathrm{dV}+\int_{\Omega} h\left(u_{\alpha}\right) \mathrm{dV}\right)^{\left(n^{2}-n-1\right) / n}
\end{aligned}
$$

where $h\left(u_{\alpha}\right)=\left(\operatorname{sign}\left(u_{\alpha}\right)\right)\left|u_{\alpha}\right|^{n^{2} /\left(n^{2}-n-1\right)}$. We already proved that $\int_{M}|\nabla v|^{n} \mathrm{dV} \leq$ $C$. Since $M=\Omega_{1} \cup \Omega \cup \Omega_{-1}$,

$$
\int_{\Omega_{1} \cup \Omega_{-1}} u_{\alpha} \mathrm{dV}+\int_{\Omega} h\left(u_{\alpha}\right) \mathrm{dV}=\int_{M} u_{\alpha} \mathrm{dV}-\int_{\Omega} u_{\alpha} \mathrm{dV}+\int_{\Omega} h\left(u_{\alpha}\right) \mathrm{dV}
$$


Since $u_{\alpha} \in H, \int_{M} u_{\alpha} \mathrm{dV}=0$, and since on $\Omega,\left|u_{\alpha}\right| \leq 1$ we get $\int_{\Omega} h\left(u_{\alpha}\right) \mathrm{dV} \leq C$ and this way

$$
\left[\int_{\Omega_{1}} u_{\alpha}^{p\left(n^{2}-n-1\right) / n^{2}} \mathrm{dV}-\int_{\Omega_{-1}}\left|u_{\alpha}\right|^{p\left(n^{2}-n-1\right) / n^{2}} \mathrm{dV}+\int_{\Omega} u_{\alpha}^{p} \mathrm{dV}\right]^{n / p} \leq C
$$

With $p=n^{3} /\left(n^{2}-n-1\right)$ we get

$$
\int_{M} u_{\alpha}^{n} \mathrm{dV}-\int_{\Omega} u_{\alpha}^{n} \mathrm{dV}+\int_{\Omega} u_{\alpha}^{n^{3} /\left(n^{2}-n-1\right)} \mathrm{dV} \leq C
$$

With the same argument that on $\Omega\left|u_{\alpha}\right| \leq 1$, we get that $\int_{M} u_{\alpha}^{n} \mathrm{dV} \leq C$, which is the contradiction we are looking for, according to $(\star)$. The theorem is proved.

\section{References}

1. Aubin, Th.: Espaces de Sobolev sur les variétés Riemanniennes. Bull. Sc. Math. 100, 149-173 (1976)

2. Aubin, Th.: Meilleures constantes dans le théorème d'inclusion de Sobolev et un théorème de Fredholm non linéaire pour la transformation conforme de la courbure scalaire, J. Funct. Anal. 32, 148-174 (1979)

3. Aubin, Th., Cotsiolis, A.: Equations elliptiques non linéaires sur $S_{n}$ dans le cas supercritique. Bull. Sc. Math. 123, 33-45 (1999)

4. Cherrier, : Une inégalité de Sobolev sur les variétés Riemanniennes. Bull. Sc. Math. 103, 353-374 (1979)

5. Collion, S.: Fonctions critiques et EDP elliptiques sur les variétés riemanniennes. Preprint de l'IEC Nancy, 2003

6. Djadli, Z., Druet, O.: Extremal functions for optimal Sobolev inequalities on compact manifolds. Calc. Var. 12, 59-84 (2001)

7. Druet, O.: The best constants problem in Sobolev inequalities. Math. Ann. 314, $327-$ 346 (1999)

8. Druet, O.: Optimal Sobolev inequalities of arbitrary order on compact riemannian manifolds. J. Funct. Anal. 159, 217-242 (1998)

9. Druet, O., Hebey, E., Vaugon, M.: Sharp Sobolev-Poincare inequalities on compact Riemannian manifolds. Trans. Am. Math. Soc. 354, 1193-1213 (2002)

10. Druet, O., Robert, F.: Asymptotic profile for the sub-extremals of the sharp Sobolev inequality on the sphere. Commun. Part. Diff. Eq. 26, 743-778 (2001)

11. Druet, O., Robert, F.: Asymptotic profile and blow-up estimates on compact Riemannian manifolds. Prépublication de L'Université de Cergy-Pontoise, 2000

12. Faget, Z.: Best constants in Sobolev inequalitites on Riemannian manifolds in the presence of symmetries. Pot. Anal. 17, 105-124 (2002)

13. Faget, Z.: Optimal constants in critical Sobolev inequalities on Riemannian manifolds in the presence of symmetries. Ann. Global Anal. Geometry 24, 161-200 (2003)

14. Hebey, E.: Fonctions extrémales pour une inégalité de Sobolev optimale dans la classe conforme de la sphère. J. de Mathématiques Pures et Appliquées 77, 721-733 (1998)

15. Hebey, E.: Nonlinear analysis on manifolds: Sobolev spaces and inequalities. Courant Institute of Mathematical Sciences Lecture Notes in Mathematics 5, 1999 
16. Hebey, E., Vaugon, M.: Meilleures constantes dans les théorèmes d'inclusion de Sobolev et multiplicité pour les problèmes de Niremberg et Yamabe. In. Univ. Math. J. 41, 377-407 (1992)

17. Hebey, E., Vaugon, M.: The best constant problem in the Sobolev imbedding theorem for complete Riemannian manifolds. Duke Math. J. 79, 235-279 (1995)

18. Hebey, E., Vaugon, M.: Meilleures constantes dans le théorème d'inclusion de Sobolev. Ann. Inst. Henri Poincaré, Analyse non-linéaire 13, 57-93 (1996)

19. Hebey, E., Vaugon, M.: Sobolev spaces in the presence of symmetries. J. Math. Pures Appl. 76, 859-881 (1997)

20. Humbert, E.: Extremal functions for the L2-Nash inequality. Preprint de l'université de Cergy-Pontoise, 1999

21. Spivak, M.: A comprehensive introduction to differential geometry, vol 1, 1979

22. Trudinger, N.: On Harnack type inequalities and their applications to quasilinear elliptic equations. Commun. Pure App. Math. 20, 721-747 (1967) 